\title{
Thermalization after/during reheating
}

\section{Keisuke Harigaya ${ }^{a}$ and Kyohei Mukaida ${ }^{b}$}

${ }^{a}$ Kavli Institute for the Physics and Mathematics of the Universe (WPI), University of Tokyo, Kashiwa, Chiba 27\%-8583, Japan

${ }^{b}$ Department of Physics, Faculty of Science, University of Tokyo, Bunkyo-ku, Tokyo 133-0033, Japan

E-mail: keisuke.harigaya@ipmu.jp, mukaida@hep-th.phys.s.u-tokyo.ac.jp

ABSTRACT: If reheating of the Universe takes place via Planck-suppressed decay, it seems that the thermalization of produced particles might be delayed, since they have large energy/small number densities and number violating large angle scatterings which decrease the momentum of particles by large amount are inefficient correspondingly. In this paper, we study the thermalization of such "under occupied" decay products in detail, following recent developments in understanding the thermalization of non-abelian plasma. Contrary to the above naive expectation, it is shown that in most cases thermalization after/during reheating occurs instantaneously by properly taking account of scatterings with small angles and of particles with small momenta. In particular, the condition for instantaneous thermalization before the completion of reheating is found to be $\alpha^{8 / 5} \gg\left(m_{\phi} / M_{\mathrm{pl}}\right)\left(M_{\mathrm{pl}}^{2} \Gamma_{\phi} / m_{\phi}^{3}\right)^{1 / 5}$, which is much milder than that obtained in previous works with small angle scatterings taken into account.

Keywords: Cosmology of Theories beyond the SM, Thermal Field Theory

ARXIV EPRINT: 1312.3097 


\section{Contents}

1 Introduction 1

2 Brief review of the reheating process 3

3 First look at thermalization processes: effects of large angle scatterings 4

4 Closer look at thermalization processes $\quad 6$

$\begin{array}{ll}4.1 \text { Basic ingredients } & 7\end{array}$

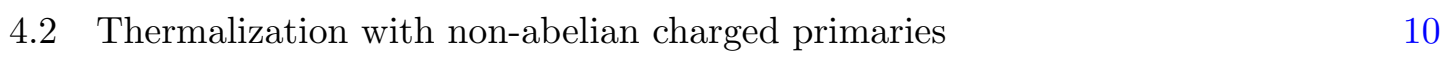

5 Application to reheating process $\quad 13$

$\begin{array}{lll}5.1 \text { After reheating } & 13\end{array}$

$\begin{array}{lll}5.2 & \text { During reheating } & 14\end{array}$

6 Conclusion and discussions $\quad 15$

\section{Introduction}

Thermodynamics plays a central role in the standard cosmology. The early Universe is much denser than the present one and hence is filled with fluid of plasma, whose dynamics is described by thermodynamics and simply characterized by a temperature. This picture is strongly supported by the observation of the cosmic microwave background $[1,2]$ and the success of the big-bang neucleosynthesis (BBN) [3].

Tracing back the history of the Universe to the past, we encounter an era in which the energy density of the Universe is dominated by a potential energy of a scalar field, such as an inflaton [4-6], a curvaton [7-10], and a moduli field [11-13], and hence the Universe is expected to be far from thermal equilibrium. The scalar field should eventually decay and yield its energy to radiation. This process is reffered to as "reheating".

Here, a fundamental question arises. Does radiation thermalize soon after reheating? ${ }^{1}$ This consideration is necessary in order to determine whether or not radiation is simply characterized by the temperature and hence is practically important for particle cosmology. ${ }^{2}$ For example, the thermal leptogenesis [14] requires a temperature larger than $10^{9} \mathrm{GeV}[15]$, the gravitino abundance depends on the temperature of the Universe [16, 17], and the efficiency of the Affleck-Dine mechanism [18] might depend on the property of thermal plasma [19-22].

\footnotetext{
${ }^{1}$ Throughout this paper, "thermalization" means that the momentum distribution of particles are given by chemical equilibrium ones.

${ }^{2}$ Otherwise, the property of radiation strongly depends on its initial conditions.
} 
Thermalization of radiation after reheating has been investigated in the literature. It is sometimes argued that thermalization is reached well after reheating in certain cases when the decay of the scalar field is small, ${ }^{3}$ for example if it is induced by Planck-suppressed interactions. In this case, the produced plasma is energetic but has a small number density compared with thermal one, and hence inelastic number violating processes play crucial roles. In the literature [23-26], thermalization is assumed to occur by accumulations of large angle scatterings, or, hard processes. Then, their interaction rates are suppressed by the large energy scale, which is around the mass of the scalar field (e.g., inflaton), and as a result it is concluded that thermalization might be delayed.

However, there is another limit: number violating scatterings with small angles. Then, their interaction rates are enhanced though energy loss per one event is suppressed. In refs. [27, 28], it is suggested that the enhanced small angle scatterings play important roles in thermalization. It is concluded that thermalization is completed instantaneously after reheating as long as the mass of the scalar field is smaller than $10^{13} \mathrm{GeV}$ [28].

At the same time, in the context of the quark gluon plasma (QGP), more thorough study is performed with hot QCD theories while paying attentions to effects of small angle scatterings and to the evolution of particles with small momenta, or, soft processes [29, 30]. Importantly, it is pointed out that themalzation is dominated by soft processes in the case of sparse number density, and thermalization proceeds from a soft sector, that is, so-called "bottom-up thermalization" takes place [29].

In this paper, we follow and generalize the discussion in ref. [30], and estimate thermalization time scales by gauge interactions. We consider a thermalization process after/during reheating while paying attention to the red-shift of momenta and the dilution of the number density by a cosmic expansion, and provide with conditions under which radiation thermalizes soon after they are emitted from the scalar field. We show that thermalization after/during reheating occurs instantaneously in most cases within the cosmic time scale by properly taking small angle scatterings and the evolution of the soft sector into account. In particular, it is found that the condition for instantaneous thermalization, $\alpha^{8 / 5} \gg\left(m_{\phi} / M_{\mathrm{pl}}\right)\left(M_{\mathrm{pl}}^{2} \Gamma_{\phi} / m_{\phi}^{3}\right)^{1 / 5}$, is much milder than that obtained in ref. [28], since the growth of the soft sector promotes the energy dissipation of decay products with large momenta. As a result, it is found that thermalization is completed instantaneously even if the mass of the scalar field is as large as $10^{15} \mathrm{GeV}$, which is the case with inflation models discussed in refs. [31-34].

This paper is organized as follow. In section 2, we briefly review how reheating proceeds. In section 3, a thermalization process solely by large angle scatterings is considered. In section 4, we discuss how thermalization proceeds and estimate thermalization time scales by taking soft processes into account, following ref. [30]. Then, thermalization after/during reheating is studied in section 5. The last section is devoted to conclusion and discussions.

\footnotetext{
${ }^{3} \mathrm{~A}$ small decay width is expected in order to suppress quantum corrections to the inflaton potential [27].
} 


\section{Brief review of the reheating process}

In this section, we review a reheating process in which an oscillating scalar field $\phi(t)$ loses its energy through a friction by the expansion of the Universe (the Hubble friction) and decays into lighter particles. Throughout this paper, we concentrate on the case in which the scalar field dominates the energy density of the Universe and the decay is well described by a perturbative decay. ${ }^{4}$

In the expanding Universe, the energy density of the oscillating scalar field $\rho_{\phi}$ and that of the decay products $\rho_{r}$ follow the equations of motion,

$$
\begin{aligned}
\frac{\mathrm{d}}{\mathrm{d} t} \rho_{\phi}+\left(3 H+\Gamma_{\phi}\right) \rho_{\phi} & =0, \\
\frac{\mathrm{d}}{\mathrm{d} t} \rho_{r}+4 H \rho_{r}-\Gamma_{\phi} \rho_{\phi} & =0, \\
3 H^{2} M_{\mathrm{pl}}^{2} & =\rho_{\phi}+\rho_{r},
\end{aligned}
$$

where $H$ and $\Gamma_{\phi}$ are the Hubble parameter of the Universe and the decay rate of the scalar field, respectively. Here, we have assumed that the self interaction of the scalar field is negligible, which is a good approximation in the last stage of the oscillation. We have also assumed that the decay products are relativistic particles.

At the early stage of the oscillation, the energy density of the Universe is dominated by the scalar field and hence the Hubble parameter is given by $H=\sqrt{\rho_{\phi}} /\left(\sqrt{3} M_{\mathrm{pl}}\right)$. Also, the decay width is negligible in comparison with the Hubble parameter. Within this approximation, the solution of eq. (2.1)-(2.3) is given by

$$
\rho_{\phi} \simeq \frac{4}{3} \frac{M_{\mathrm{pl}}^{2}}{t^{2}}, \quad \rho_{r} \simeq \frac{4}{5} \Gamma_{\phi} M_{\mathrm{pl}}^{2} \frac{1}{t} \sim \rho_{\phi} \Gamma_{\phi} t, \quad H=\frac{2}{3 t} .
$$

The energy density of the radiation is dominated by particles with momentum $p \sim m_{\phi}$, and hence the distribution function at that momentum is given by

$$
f\left(p \sim m_{\phi}\right) \sim \frac{\rho_{r}}{m_{\phi}^{4}} \sim \frac{\Gamma_{\phi} M_{\mathrm{pl}}^{2}}{m_{\phi}^{4} t}
$$

for each time. Note that the scalar field produces particles with $p \sim m_{\phi}$ and particles with lower momenta are provided by the red-shift. Since the momentum is red-shifted in proportion to $t^{-2 / 3}$ during a matter dominated era, the distribution function is given by [25]

$$
f(p) \sim \begin{cases}\left(\frac{\Gamma_{\phi} M_{\mathrm{pl}}^{2}}{m_{\phi}^{3}}\right)\left(m_{\phi} t\right)^{-1}\left(\frac{m_{\phi}}{p}\right)^{3 / 2} & \text { for }\left(t / t_{i}\right)^{-2 / 3} m_{\phi} \lesssim p \lesssim m_{\phi} \\ 0 & \text { for otherwise }\end{cases}
$$

\footnotetext{
${ }^{4}$ If the oscillation time scale of the scalar field is much slower than the typical interaction time scale of background plasma, the interactions of the decay products of the scalar field with the radiation affect the dissipation process [35-41], and the discussion on the thermalization process strongly depends on the detail of the theory.
} 
if the produced particles do not interact at all. Here, $t_{i}$ is the time at which the oscillation of the scalar field begins to dominate the energy density of the Universe. ${ }^{5}$

The energy densities of the scalar field and of the radiation become comparable at $t^{-1} \sim H \sim \Gamma_{\phi}$. After this point, the Universe is radiation-dominant. This point is referred to as "the end of reheating", and the "reheating temperature" $T_{\mathrm{rh}}$ is often defined by

$$
3 \Gamma_{\phi}^{2} M_{\mathrm{pl}}^{2} \equiv \frac{\pi^{2}}{30} g_{*} T_{\mathrm{rh}}^{4},
$$

where $g_{*}$ is the effective degree of freedom of would-be thermalized particles. If the decay products of the scalar field interact with each others sufficiently fast, they thermalize soon after the end of reheating. $T_{\mathrm{rh}}$ coincides with the temperature of the Universe at that point. In the following sections, we discuss how thermalization proceeds by interactions among decay products.

\section{First look at thermalization processes: effects of large angle scatterings}

In this section, we consider a thermalization process solely by large angle scatterings, as is assumed in refs. [23-26]. Here, "large angle" means that the scatterings involve large momentum exchanges so that particles lose their energy efficiently. We will see that large angle scatterings cannot bring about instantaneous thermalization after reheating, if the decay rate of the scalar field is small, such as the one induced by Planck-suppressed interactions. The result shows the necessity of consideration of thermalization processes with cares.

In order to investigate whether thermalization occurs soon after the end of reheating, let us first estimate the number density of the decay products. Just after the end of reheating, the number density of the decay products, $n_{i}$, is as large as

$$
\left.n_{i} \simeq \frac{\rho_{\phi}}{m_{\phi}}\right|_{t \sim \Gamma_{\phi}^{-1}} \simeq \frac{\Gamma_{\phi}^{2} M_{\mathrm{pl}}^{2}}{m_{\phi}},
$$

where we have used the result obtained in the previous section. As we have mentioned in the previous section, the momentum of decay products, $p_{i}$, is as large as the mass of the scalar field, $p_{i} \simeq m_{\phi}$.

Let us compare this number density with a thermal one. If thermalization occurs instantaneously, number densities of particles, $n_{\mathrm{th}}$, are as large as

$$
n_{\mathrm{th}} \simeq T_{\mathrm{rh}}^{3} \simeq \Gamma_{\phi}^{3 / 2} M_{\mathrm{pl}}^{3 / 2}
$$

Its ratio to $n_{i}$ is given by

$$
\frac{n_{\mathrm{th}}}{n_{i}} \simeq \frac{m_{\phi}}{\sqrt{\Gamma_{\phi} M_{\mathrm{pl}}}}
$$

\footnotetext{
${ }^{5}$ Below the infrared edge, there is a contribution from the decay of the scalar field before its oscillation dominates the energy density of the Universe in general. However, the shape of the contribution is always shallower than $p^{-3 / 2}$. Further, as we will see later, the time scale of thermalization is governed by the distribution of the hardest particles. Therefore, we neglect such infrared contributions in the following.
} 
For example, let us consider two extreme cases, in which the decay occurs due to an order one Yukawa coupling and a dimension 5 Planck-suppressed interaction. Decay rates are as large as

$$
\Gamma_{\phi} \simeq\left\{\begin{array}{ll}
m_{\phi} & \text { (Yukawa }) \\
m_{\phi}^{3} / M_{\mathrm{pl}}^{2} & \text { (dimension 5 Planck-suppressed) }
\end{array} .\right.
$$

Accordingly, the ratio of the number densities is given by

$$
n_{\mathrm{th}} / n_{i} \simeq\left\{\begin{array}{ll}
\sqrt{m_{\phi} / M_{\mathrm{pl}}} \ll 1 & \text { (Yukawa) } \\
\sqrt{M_{\mathrm{pl}} / m_{\phi}} \gg 1 & \text { (dimension 5 Planck-suppressed) }
\end{array} .\right.
$$

Therefore, if the decay occurs via the order one Yukawa coupling, the number density is larger than the thermalized one, in other words, the Universe is over occupied. On the other hand, if the decay occurs via the Planck-suppressed interaction, the number density is smaller than the thermalized one, in other words, the Universe is under occupied.

For the over occupied case, $T_{\mathrm{rh}}$ is larger than $m_{\phi}$. In such a case, it is known that thermal effects play important role in the reheating process [42, 43], and the resulting tempetarure of the Universe depends on the detail of the theory. ${ }^{6}$ Throughout this paper, we assume the under occupied case, in which the decay of the scalar field is described by the field theory in the zero temperature.

As we have declared at the beginning of this section, let us consider large angle scatterings. In order to achieve thermalization, scatterings which increase the number density of the particles are necessary. The most efficient ones would be two body to three body scatterings. Cross sections of such scatterings, $\sigma_{i}$, are as large as

$$
\begin{aligned}
\sigma_{i} & \simeq k \frac{1}{p_{i}^{2}} \simeq k \frac{1}{m_{\phi}^{2}}, \\
k & \simeq \frac{g^{6}}{128 \pi^{3}},
\end{aligned}
$$

where $g$ is a gauge or Yukawa coupling constant. For example, QCD interactions yield $k \simeq 10^{-3}$. Therefore, just after the end of reheating, an average interaction rate is given by

$$
\Gamma_{\mathrm{int}} \simeq\langle\sigma n v\rangle \simeq \sigma_{i} n_{i} \simeq k \frac{\Gamma_{\phi}^{2} M_{\mathrm{pl}}^{2}}{m_{\phi}^{3}}
$$

A ratio between the interaction rate and the Hubble parameter just after the end of reheating is given by

$$
\frac{\langle\sigma n v\rangle}{H} \simeq k \frac{\Gamma_{\phi} M_{\mathrm{pl}}^{2}}{m_{\phi}^{3}}
$$

This ratio is larger than one if

$$
\Gamma_{\phi}>\frac{1}{k} \frac{m_{\phi}^{3}}{M_{\mathrm{pl}}^{2}} \gtrsim \frac{1}{k} \times \text { (Decay width by Planck-suppressed interactions). }
$$

\footnotetext{
${ }^{6}$ For some cases, the inflaton condensation is completely broken into particles and participates in the thermal plasma.
} 
In this case, thermalization occurs just after the end of reheating solely by large angle scatterings.

Therefore, if the decay of the scalar field occurs via interactions stronger than Plancksuppressed ones, the decay products of the scalar field thermalize soon after the end of reheating. This is due to large number density of the decay products. On the other hand, if the decay takes place via Planck-suppressed interactions, thermalization does not occur soon after the end of reheating, as long as large angle scatterings are concerned.

What will happen if thermalization is controlled solely by large angle scatterings, when the decay is via Planck-suppressed interactions? As Universe expends, momenta of the decay products, number density, and the Hubble parameter decrease in proportional to $a^{-1}$, $a^{-3}$, and $a^{-2}$, where $a$ is the scale parameter of the Universe. Hence, the relation between the interaction rates and the Hubble rate increases in proportional to $a^{1}$. Therefore, thermalization occurs when the Universe becomes $k^{-1}$ times larger after the end of the reheating process. The thermalized temperature $T_{\text {th }}$ is as large as

$$
T_{\mathrm{th}} \simeq k T_{\mathrm{rh}},
$$

which is smaller than $T_{\text {rh }}$ even if the QCD interaction is involved. If the scalar field does not decay into particles with the QCD interaction, $T_{\text {th }}$ would be far smaller than $T_{\text {rh }}$.

\section{Closer look at thermalization processes}

In the previous section, the simple analysis has revealed that large angle scatterings alone cannot thermalize the particles produced by the decay of the scalar field instantaneously for Planck-suppresed decay. However, in this section, we will see that actual thermalization occurs much faster with a closer look at thermalization processes, following the discussion in ref. [30]. See also ref. [44]. This is because soft particles radiated from decay products with large momenta play important roles in thermalization, which is neglected in the previous section. Consequently, as we will see in section 5, thermalization after reheating occurs rather instantaneously even for Planck-suppressed decay.

First, let us recall the discussion in section 2. We have shown that at each point during and after reheating, although the energy and number densities of decay products are dominated by particles with momenta $p \sim m_{\phi}$, their distribution becomes complicated due to the red-shift of previously produced particles: eq. (2.6). As a first step, it is instructive to neglect the expansion of the Universe and consider the case in which the distribution function is given by a mono-spectrum one:

$$
f(p) \simeq \begin{cases}n_{h} / m_{\phi}^{3} & \text { for } p \sim m_{\phi} \\ 0 & \text { for others }\end{cases}
$$

where $n_{h}$ is the number density of the decay products with large momenta, which we call the "hard primaries". Effects of previously produced particles are separately discussed in section 5.2. There, we find that the effects of the previously produced particles are negligible in estimating the thermalization time scale and hence the mono-spectrum approximation is an appropriate description. 
Throughout this paper, we assume that the decay products interact with each other by gauge interactions. Following ref. [30], the distribution is expressed by the fine structure constant of a gauge interaction $\alpha \equiv g^{2} /(4 \pi)$ as

$$
\alpha^{-c} \equiv n_{h} / p_{i}^{3},
$$

where $p_{i}=m_{\phi}{ }^{7}$ Cases with $c>0$ are referred to as over occupied systems and those with $c<0$ are referred to as under occupied systems, and processes of thermalization are found to be different for each cases [30].

As we have mentioned in the previous section, we are particularly interested in the case with

$$
\alpha^{-c}=\frac{\Gamma_{\phi}^{2} M_{\mathrm{pl}}^{2}}{m_{\phi}^{4}},
$$

which typically implies $c<0$ for the Planck-suppressed decay. ${ }^{8}$ Hence, let us concentrate on the under occupied case defined as $c<0$ in the following.

\subsection{Basic ingredients}

Before reviewing thermalization in the under occupied case, let us briefly summarize basic ingredients for the themalization process [30, 44]. Here, we consider thermalization by a gauge interaction, which is effective due to t-channel enhancement as we will see later. In the medium, the dispersion relation of particle-like excitation, so-called quasi-particle, can be approximated with

$$
\omega(\boldsymbol{p}) \simeq \sqrt{\boldsymbol{p}^{2}+m_{s}^{2}} ; \quad m_{s}^{2} \sim \alpha \int_{\boldsymbol{p}} \frac{f(p)}{p},
$$

where $\int_{\boldsymbol{p}} \equiv \int d^{3} p /(2 \pi)^{3}, m_{s}$ is the screening mass, and $f(p)$ is the distribution function of the "screener", which interacts with the quasi-particle.

The non-equilibrium dynamics of plasma can be studied by the Boltzmann equations of quasi-particles with collision terms of spatially localized interactions which can be obtained from $S$-matricies, if the system respects the following two conditions: (i) the typical size of quasi-particles is smaller than the mean free path, (ii) the typical duration of interaction is shorter than the mean free time. Note that when the Landau Pomeranchuk Migdal (LPM) effect [45-50] becomes efficient, the condition (ii) does not hold and hence we need special cares as we will see later. If both (i) and (ii) are met, the distribution function $f(t, \boldsymbol{p})$ obeys the Boltzmann equation:

$$
\left[\partial_{t}-H \boldsymbol{p} \cdot \frac{\partial}{\partial \boldsymbol{p}}\right] f(t, p)=-\mathcal{C}[f]
$$

where $H$ is the Hubble parameter and $\mathcal{C}$ represents a collision term via spatially local interactions. Here we gave restricted ourselves to an isotropic system which we are interested in.

\footnotetext{
${ }^{7}$ The following discussion is a general one and the assumption that $p_{i}=m_{\phi}$ is not necessary.

${ }^{8}$ For instance, $\alpha^{-c} \sim 10^{-10}\left(m_{\phi} / 10^{13} \mathrm{GeV}\right)^{2}$ for the dimension 5 Planck-suppressed decay.
} 


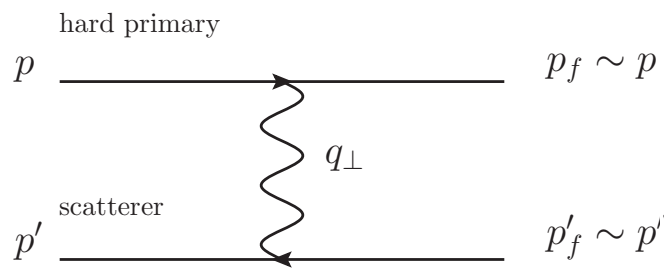

Figure 1. Two-to-two scattering.

The collision term $\mathcal{C}$ imprints basic elementary processes: number conserving two-to-two scattering and number violating nearly collinear in-medium splitting.

The former process (figure 1) diffuses the momentum of particles and the average of squared momentum transfer is described by a random walk:

$$
(\Delta p)^{2} \sim \hat{q}_{\mathrm{el}} t
$$

with $\hat{q}_{\text {el }}$ being a diffusion constant that is estimated as

$$
\hat{q}_{\mathrm{el}} \sim \int d^{2} q_{\perp} \frac{d \Gamma_{\mathrm{el}}}{d q_{\perp}^{2}} q_{\perp}^{2} \sim \alpha^{2} \int_{p^{\prime}} f\left(p^{\prime}\right)\left[1 \pm f\left(p^{\prime}\right)\right],
$$

where $f\left(p^{\prime}\right)$ is the distribution function of "scatterers", which scatter the hard primaries off. The sign $+/-$ corresponds to the case in which the scatterers are bosonic/fermionic. Here $\Gamma_{\text {el }}$ is an elastic scattering rate with a momentum exchange $q_{\perp}<p$, where $q_{\perp}$ is a momentum transfer.

For example, the scattering by the t-channel exchange of the gauge boson yields

$$
\frac{d \Gamma_{\mathrm{el}}}{d q_{\perp}^{2}} \sim \frac{\alpha^{2}}{q_{\perp}^{2}\left(q_{\perp}^{2}+m_{s}^{2}\right)} \int_{p^{\prime}} f\left(p^{\prime}\right)\left[1 \pm f\left(p^{\prime}\right)\right] .
$$

Due to enhancement for small $q_{\perp}$, this scattering, if it exists, plays important roles. ${ }^{9}$ In fact, the t-channel enhanced soft elastic scatterings occur so frequently that the cosmic expansion can be neglected: $H \ll \Gamma_{\mathrm{el}}^{(\text {soft })} \sim \hat{q}_{\mathrm{el}} / m_{s}^{2}$. Therefore, we neglect the cosmic expansion at first and compare the time scale of bottleneck process with that of cosmic expansion finally.

On the other hand, since the soft elastic scattering with $q_{\perp} \sim m_{s}$ makes the quasiparticle slightly off-shell in the medium (in-medium width), the latter process change the number by radiating a nearly collinear gauge boson (figure 2). As mentioned earlier, if each soft elastic scatterings occur more frequently than the duration of the splitting process, the condition (ii) is violated and one has to take the quantum mechanical interference of each scatterings into account, that is, we have to care about the LPM effect [45-50].

Let us estimate the time scale of quantum interference. Suppose that the parent particle travels through a spatial distance $\boldsymbol{x}=t \hat{\boldsymbol{x}}$ with a time interval $t[x=(t, \boldsymbol{x})]$ under soft elastic scatterings. The interference of bremsstrahlung produced by the first and last

\footnotetext{
${ }^{9}$ This enhancement is absent for the t-channel exchange of a scalar particle by a Yukawa interaction.
} 


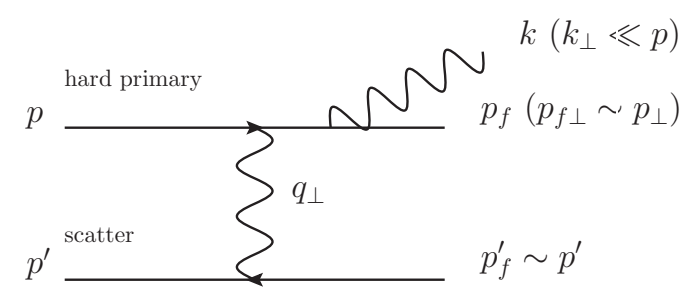

Figure 2. One example of collinear in-medium splittings without the LPM effect.

scatterings, at $\mathbf{0}$ and $\boldsymbol{x}$, remains until the phase factor varies significantly, $1 \gtrsim k \cdot x \sim t k \theta^{2}$, with $k$ being the momentum of the daughter particle and $\theta$ being the emission angle of the daughter particle.

When the daughter particle is a non-abelian gauge field, it is also charged and is subjected to the soft elastic scatterings. The coherence is lost if the daughter particle changes its direction substantially due to these random soft collisions, $1 / \sqrt{k t} \sim \theta\left(\sim k_{\perp} / k\right)$. Recalling that the perpendicular momentum diffuses via elastic scatterings as $k_{\perp}^{2} \sim \hat{q}_{\mathrm{el}} t$, one finds the quantum mechanical formation time scale as ${ }^{10}$

$$
t_{\mathrm{form}}^{\mathrm{NA}} \sim \max \left[1 / \Gamma_{\mathrm{el}}^{(\mathrm{soft})}, \sqrt{k / \hat{\mathrm{q}}_{\mathrm{el}}}\right]
$$

where $\Gamma_{\mathrm{el}}^{\text {(soft) }}$ is the rate of one soft elastic scattering $q_{\perp} \sim m_{s}$, which is given by $\Gamma_{\mathrm{el}}^{\text {(soft) }} \sim$ $\hat{q}_{\mathrm{el}} / m_{s}^{2}$. Then, the rate of the collinear splitting in the medium is roughly estimated as

$$
\Gamma_{\text {split }}^{\mathrm{NA}}(k) \sim \alpha t_{\text {form }}^{\mathrm{NA}^{-1}}(k)
$$

with $k$ being the momentum of the daughter particle. Note that the LPM effect becomes efficient when the momentum $k$ exceeds a typical value:

$$
k_{\mathrm{LPM}}^{\mathrm{NA}} \equiv m_{s}^{4} / \hat{q}_{\mathrm{el}} .
$$

When the daughter particle is an abelian gauge field, however, it is not charged under its own gauge group. Therefore, the coherence remains unless the parent particle changes its direction substantially, $\theta \sim p_{\perp} / p \sim 1 / \sqrt{k t}$ with $p$ being the momentum of the parent particle. Contrary to the non-abelian case, the formation time scale depends on the momentum of the parent particle as

$$
t_{\text {form }}^{\mathrm{U}(1)} \sim \max \left[1 / \Gamma_{\mathrm{el}}^{(\mathrm{soft})}, \sqrt{p^{2} /\left(k \hat{q}_{\mathrm{el}}\right)}\right]
$$

Note that the LPM effect dominates the collinear splitting when the momentum $\mathrm{k}$ becomes smaller than a typical value:

$$
k_{\mathrm{LPM}}^{\mathrm{U}(1)} \equiv \hat{q}_{\mathrm{el}} Q^{2} / m_{s}^{4} .
$$

\footnotetext{
${ }^{10}$ Note that at least one soft elastic scattering is required for the collinear splitting to occur.
} 

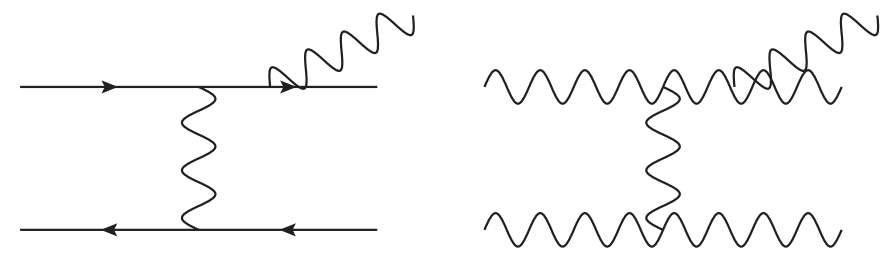

Figure 3. Examples of creation of soft population by collinear splittings for the non-Abelian case without the LPM effect.

\subsection{Thermalization with non-abelian charged primaries}

We are now in a position to discuss how thermalization proceeds. In the following, we consider the case in which the majority of primary particles produced from the decay of the scalar field is charged under some non-abelian gauge group or non-abelian gauge bosons themselves. We consider that this case is practically most important. ${ }^{11}$ The thermalization of QCD is discussed in ref. [30] and we closely follow the discussion there. As we will see, soft particles produced by collinear splittings via small angle scatterings play crucial roles in thermalization after/during reheating.

First of all, soft bosons are radiated from the primary hard particles with the momentum $p_{i} \sim m_{\phi}$ via collinear splittings in association with t-channel enhanced soft elastic scatterings, whose rate is given in eq. (4.8), and a new population around small momentum is created (figure 3). The phase space distribution of these soft particles is estimated as

$$
f_{s}(t, p) \sim \Gamma_{\mathrm{split}}^{\mathrm{NA}}(p) n_{h} p^{-3} t \sim \begin{cases}\alpha \Gamma_{\mathrm{el}}^{(\mathrm{soft})} n_{h} p^{-3} t & \text { for } p<k_{\mathrm{LPM}}^{\mathrm{NA}} \\ \alpha \sqrt{\hat{q}_{\mathrm{el}}} n_{h} p^{-7 / 2} t & \text { for } k_{\mathrm{LPM}}^{\mathrm{NA}}<p\end{cases}
$$

For later convenience, let us summarize quantities determined by the number density of the hard primaries:

$$
m_{s}^{2} \sim \alpha^{1-c} p_{i}^{2}, \quad \hat{q}_{\mathrm{el}} \sim \alpha^{2-c} p_{i}^{3}, \quad k_{\mathrm{LPM}}^{\mathrm{NA}} \sim \alpha^{-c} p_{i}\left(<m_{\phi}\right) .
$$

Note that the spectrum is quite sharp compared with a thermal-like distribution, that is proportional to $p^{-1}$ and hence the "soft sector" is effectively over occupied. They interact among themselves and with hard particles, and fall into a thermal-like distribution below a scale $p_{\max }$ :

$$
f_{s} \sim T_{*} / p ; \text { for } p<p_{\max } .
$$

The typical scale $p_{\max }$ evolves towards a UV regime via interactions not only among soft particles but with the remaining hard particles. Let us estimate the typical time scale of $p_{\max }$ evolution. In the following, let us consider the case with $m_{s}<k_{\mathrm{LPM}}$, that is $c>-1$. For $c<-1$, the screening mass is larger than $k_{\mathrm{LPM}}$ from the beginning and

\footnotetext{
${ }^{11}$ For example, as long as the decay of the scalar field respects the grand unified theory, the majority of the decay products always has non-abelian gauge interactions. The case with an abelian gauge interaction is discussed elsewhere [51]. There, we find that thermalization proceeds slower than the case of the non-abelian gauge interaction. This is because the gauge boson, which plays an important role in the thermalization process as we will see, is not charged under its own gauge group in abelian gauge theories.
} 
the production of soft particles is always LPM suppressed. In this case, one can skip the following discussion until eq. (4.24). The important processes that drive $p_{\max }$ towards UV are elastic scatterings with hard particles and saturation between the production and absorption of soft particles. The former process leads to the following growth rate of $p_{\text {max }}$ :

$$
p_{\max }^{\text {(h scat) }} \sim \sqrt{\hat{q}_{\mathrm{el}}^{(\text {hard })} t} \sim \alpha^{1-c / 2} p_{i} \sqrt{p_{i} t} .
$$

The latter process is characterized by the balance of production and absorption rate for the soft population:

$$
\begin{aligned}
0 & =\int_{\boldsymbol{p}} \Gamma_{\text {split }}\left[f(p)(1+f(p-k))\left(1+f_{s}(k)\right)-f(p-k) f_{s}(k)(1+f(p))\right] \\
& \sim \int_{\boldsymbol{p}} \Gamma_{\text {split }}\left[k f^{\prime}(p) f_{s}(k)+f(p)\right]
\end{aligned}
$$

which implies

$$
f_{s}(k) \sim \frac{\int_{\boldsymbol{p}} f(p)}{\int_{\boldsymbol{p}}-k f^{\prime}(p)} \sim \frac{p_{i}}{k} .
$$

Hence, if the distribution of soft modes exceeds $f_{s}(k) \sim p_{i} / k$, then the production of soft particles is cancelled by the absorption. By equating this condition with eq. (4.14), one finds the typical scale below which the distribution turns into the thermal-like one:

$$
p_{\max }^{(\mathrm{sat})} \sim \alpha^{1-c / 2} p_{i} \sqrt{p_{i} t},
$$

that is equal to eq. (4.17). Note that the effective temperature for the soft sector is given by

$$
T_{*} \sim p_{i}
$$

that remains constant at this regime. Also note that still, the screening mass, the energy and number densities are dominated by the remaining hard particles.

As the the scale $p_{\max }$ evolves, the screening mass becomes dominated by the soft particles and also the LPM scale $k_{\mathrm{LPM}}^{\mathrm{NA}}$ becomes comparable to $p_{\max }$. This time scale is estimated as

$$
\left(p_{i} t\right)_{\mathrm{LPM}} \sim \alpha^{-2-c}
$$

After that, the production rate of soft particles given in eq. (4.10) is dominated by the LPM suppressed one, and hence the effective temperature no longer remains constant: ${ }^{12}$

$$
T_{*} \sim p_{\max }\left(\alpha \sqrt{\hat{q}_{\mathrm{el}} / p_{\max }}\right) n_{h} t / p_{\max }^{3} \sim \alpha^{-c / 4-1 / 2} p_{i}\left(p_{i} t\right)^{-1 / 4} .
$$

The soft sector "thermalizes" when the effective temperature $T_{*}$ becomes comparable to $p_{\text {max }}$, which occurs at

$$
\left(p_{i} t\right)_{\mathrm{soft} \text { th }} \sim \alpha^{-2+c / 3} .
$$

\footnotetext{
${ }^{12}$ Here, we have assumed that $\hat{q}_{\mathrm{el}}$ is determined by the hard primaries. From eq. (4.23), it can be shown that the contribution of the soft sector to $\hat{q}_{\mathrm{el}}$ is as large as that of the hard primaries.
} 
Around that time, not only the screening mass but the number density are dominated by soft particles: $n_{s} \sim T_{*}^{3} \gtrsim n_{h} \sim \alpha^{-c} p_{i}^{3}$. However, the energy density is still dominated by the remaining hard particles.

Finally, the remaining hard particles dissipate their energy to the soft "thermal" sector via multiple splitting of daughter particles. One can determine the maximum scale $k_{\text {split }}$ below which a particle can deposit an order one fraction of its energy into the background soft "thermal" sector from a criteria $\Gamma_{\mathrm{LPM}}^{\mathrm{NA}}\left(k_{\text {split }}\right) t \sim 1$. The remaining hard particles lose their energy dominantly via the splitting of "hardest" daughter particle with momentum $k_{\text {split. }}$. Through this process, the "thermal" sector is heated, and the energy conservation implies $T_{*}^{4} \sim k_{\text {split }} n_{h}$. These equations suggest

$$
\begin{aligned}
T_{*} & \sim \alpha^{4-c} p_{i}\left(p_{i} t\right)^{2}, \\
k_{\text {split }} & \sim \alpha^{-3 c+16} p_{i}\left(p_{i} t\right)^{8} .
\end{aligned}
$$

Note that time scales of processes which establish the chemical equilibrium for the soft sector is as large as

$$
\left(\alpha^{2} T_{*}\right)^{-1} \sim \alpha^{-6+c} p_{i}^{-1}\left(p_{i} t\right)^{-2},
$$

and shorter than the time scale $t$ as long as $t>t_{\text {soft }}$ th. Therefore, soon after hard particles emit daughter particles with momenta $k_{\text {split }}$ and the daughter particles deposit their energy into the soft sector, particles in the soft sector follow the chemical equilibrium. The remaining hard particles completely lose their energy when this scale becomes comparable to $p_{i}$. As a result, thermalization is completed at

$$
\left(p_{i} t\right)_{\mathrm{split}} \sim \alpha^{-2+3 c / 8}
$$

and the thermal bath with the temperature $T_{\mathrm{rh}}$ is created.

Since $c<0$, the relevant time scales satisfy the following inequality:

$$
\left(p_{i} t\right)_{\mathrm{LPM}}<\left(p_{i} t\right)_{\mathrm{soft} \text { th }}<\left(p_{i} t\right)_{\mathrm{split}} .
$$

Thus, the bottleneck process is one in which the remaining hard particles lose their energy into the background plasma, ${ }^{13}$ and hence the timescale of thermalization $t_{\mathrm{th}}$ is given by [30]

$$
t_{\mathrm{th}} \sim \frac{1}{p_{i}} \alpha^{-2+3 c / 8}
$$

Before closing this section, let us comment on abelian gauge bosons. Decay products of the scalar field often include abelian gauge bosons. Even if it is not the case, decay products in general have an abelian gauge interaction and hard primaries radiate not only non-abelian gauge bosons but also abelian gauge bosons. These hard abelian gauge bosons can dissipate their energies via collinear splittings of matters with t-channel enhancement (figure 4). Therefore, they do not affect the above discussion.

\footnotetext{
${ }^{13}$ The time scale of this final process is parametrically the same as the dissipation time scale for the hard particle with momentum $p_{i}>T_{\mathrm{rh}}$ to travel through the thermal plasma with the temperature $T_{\mathrm{rh}}$ :

$$
t_{\text {diss }} \sim\left(\alpha^{2} T_{\mathrm{rh}}\right)^{-1} \sqrt{p_{i} / T_{\mathrm{rh}}} \sim t_{\mathrm{split}} .
$$




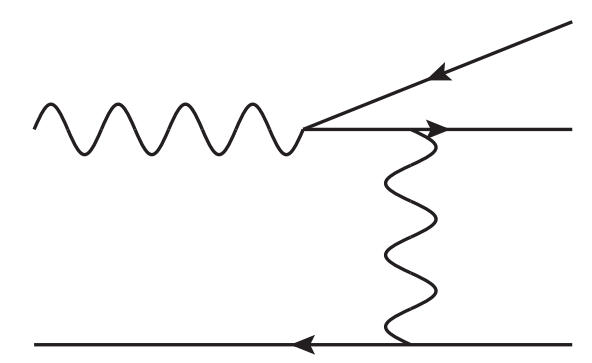

Figure 4. Example of creation of soft population by collinear splittings for the Abelian case without the LPM effect.

\section{Application to reheating process}

In this section, let us study the implication of the result given in eqs. (4.32) to the reheating process. We discuss the thermalization of decay products after and during reheating separately.

\subsection{After reheating}

At first, it is instructive to consider an instantaneous decay approximation for the scalar field. In this case, the spectrum of hard primaries is approximated with a mono-spectrum one and we can neglect effects of red-shifted previously produced particles. Actually, in the next section, we show that the effects of previously produced particles is negligible. Let us summarize the conditions where the produced particles thermalize much faster than the cosmic time scale.

Thermalization is completed against the expansion of the Universe if the times scale of the themalization is much shorter than the inverse of Hubble parameter:

$$
1 \gg H t_{\mathrm{split}} \sim \alpha^{-2}\left(\frac{m_{\phi}}{M_{\mathrm{pl}}}\right)^{3 / 4}\left(\frac{\Gamma_{\phi}}{m_{\phi}}\right)^{1 / 4}
$$

Interestingly, this inequality implies that thermalization is more likely to be completed for a smaller decay rate. Thus, in order to show that thermalization occurs instantaneously in general, it is sufficient to discuss the largest decay rate. Since we assume that the decay of the scalar field is well-described by the field theory in the zero temperature, the decay rate should be smaller than $m_{\phi}^{2} / M_{\mathrm{pl}}$. In this case, eq. (5.1) reads

$$
\alpha \gg \frac{m_{\phi}^{1 / 2}}{M_{\mathrm{pl}}^{1 / 2}}=0.002\left(\frac{m_{\phi}}{10^{13} \mathrm{GeV}}\right)^{1 / 2} .
$$

Note that the condition is severer for larger $m_{\phi}$. The largest mass of the scalar field which appears in the cosmology would be the mass of an inflaton which has a convex potential. For example, in chaotic or hybrid inflation models with convex potentials, the mass is as large as $10^{15} \mathrm{GeV}$ [31-34]. One can easily see that unless the gauge coupling is extremely suppressed, the decay products thermalize rather instantaneously compared with the cosmic time scale. As we have mentioned in the introduction, this condition is 
much milder than that obtained in ref. [28], which is given by $\alpha^{3} \gg\left(m_{\phi} / M_{\mathrm{pl}}\right)$, since the growth of the soft sector promotes the energy dissipation of hard particles.

\subsection{During reheating}

Then, let us discuss the effects of previously produced particles while following the evolution of the momentum distribution of radiation during reheating. As mentioned at the end of section 2, the scalar field decays gradually before the completion of reheating. Therefore previously produced particles are red-shifted, which results in the following distribution of hard particles [eq. (2.6)]:

$$
f_{h}(p) \sim \begin{cases}\left(\frac{\Gamma_{\phi} M_{\mathrm{pl}}^{2}}{m_{\phi}^{3}}\right)\left(m_{\phi} t\right)^{-1}\left(\frac{m_{\phi}}{p}\right)^{3 / 2} & \text { for }\left(t / t_{i}\right)^{-2 / 3} m_{\phi} \lesssim p \lesssim m_{\phi} . \\ 0 & \text { for otherwise }\end{cases}
$$

Since the number density is dominated by the hardest particles with $p \sim m_{\phi}$, the soft particles are produced dominantly from them. Hence, we can estimate the production of soft particles in the same way as section 4.2.

To avoid possible complications, we focus on the situation where the produced soft particles have enough time to thermalize against the expansion of the Universe. In order to estimate the time after which the soft sector is expected to thermalize immediately, let us recall the time scale $t_{\text {soft th }}$ which is given by eq. (4.25):

$$
\left(m_{\phi} t\right)_{\text {soft th }} \sim \alpha^{-2+c / 3} \leftrightarrow\left(m_{\phi} t\right)_{\text {soft th }} \sim \alpha^{-3}\left(\frac{m_{\phi}^{3}}{\Gamma_{\phi} M_{\mathrm{pl}}^{2}}\right)^{1 / 2} .
$$

The soft sector thermalizes within the cosmic time scale for

$$
1>t_{\mathrm{soft}} \mathrm{th} H \leftrightarrow t>t_{\mathrm{soft}} \text { th } .
$$

As discussed previously, at that time, the number density is dominated by the soft sector while the energy density is still dominantly stored in the hard sector. Note that the production of soft particles is already dominated by the LPM suppressed one.

The hard particles dissipate their energy into the soft thermal plasma via multiple splittings. As done in the previous section, we can estimate the splitting momentum $k_{\text {split }}$ below which a particle can deposit an order one fraction of its energy and participate in the thermal plasma, from the criteria: $\Gamma_{\mathrm{LPM}}^{\mathrm{NA}}\left(k_{\mathrm{split}}\right) t \sim 1$. The thermal plasma is heated by the splitting of hardest particle together with the daughter particle of the momentum $k_{\text {split }}$, since the hardest particles dominate the number density of hard sector. Hence the energy conservation gives $T_{*}^{4} \sim n_{h} k_{\text {split }}$ with $T_{*}$ being the effective temperature of soft thermal plasma. From these equations, one finds

$$
\begin{aligned}
T_{*} & \sim \alpha^{4}\left(\frac{\Gamma_{\phi} M_{\mathrm{pl}}^{2}}{m_{\phi}^{3}}\right) m_{\phi}\left(m_{\phi} t\right), \\
k_{\text {split }} & \sim \alpha^{16}\left(\frac{\Gamma_{\phi} M_{\mathrm{pl}}^{2}}{m_{\phi}^{3}}\right)^{3} m_{\phi}\left(m_{\phi} t\right)^{5} .
\end{aligned}
$$


Therefore, the distribution function of soft particles for $t>t_{\text {soft }}$ th can be roughly parametrized as

$$
f_{s}(t, p) \sim \begin{cases}\left(\frac{T_{*}}{p}\right) \cdots \text { boson or } 1 \cdots \text { fermion } & \text { for } p<T_{*} \\ \exp \left(-\frac{p}{T_{*}}\right) & \text { for } T_{*}<p<k_{\text {split }} \\ \alpha^{8}\left(\frac{\Gamma_{\phi} M_{\mathrm{pl}}^{2}}{m_{\phi}^{3}}\right)^{5 / 2}\left(m_{\phi} t\right)^{3 / 2}\left(\frac{m_{\phi}}{p}\right)^{7 / 2} & \text { for } k_{\mathrm{split}}<p<m_{\phi}\end{cases}
$$

and that of hard primaries is given by

$$
f_{h}(t, p) \sim \begin{cases}\left(\frac{\Gamma_{\phi} M_{\mathrm{pl}}^{2}}{m_{\phi}^{3}}\right)\left(m_{\phi} t\right)^{-1}\left(\frac{m_{\phi}}{p}\right)^{3 / 2} & \text { for }\left(t / t_{i}\right)^{-2 / 3} m_{\phi}<p<m_{\phi} . \\ 0 & \text { for } m_{\phi}<p\end{cases}
$$

Note that the soft sector already dominates over the red-shifted hard particles below an intermediate scale

$$
k_{\mathrm{int}} \sim \alpha^{4}\left(\frac{\Gamma_{\phi} M_{\mathrm{pl}}^{2}}{m_{\phi}^{3}}\right)^{3 / 4} m_{\phi}\left(m_{\phi} t\right)^{5 / 4}
$$

which is larger than $k_{\text {split }}$ for $t<t_{\text {split }}$ (see below) and hence the effects of red-shifted particles are not so relevant.

The remaining hard particles completely lose their energy when the splitting momentum becomes comparable to the maximum momentum: $k_{\text {split }} \sim m_{\phi}$. Consequently, thermalization is completed at

$$
\left(m_{\phi} t\right)_{\mathrm{split}} \sim \alpha^{-16 / 5}\left(\frac{\Gamma_{\phi} M_{\mathrm{pl}}^{2}}{m_{\phi}^{3}}\right)^{-3 / 5}
$$

At that time, the intermediate scale also reaches the maximum momentum simultaneously: $\left.k_{\text {int }}\right|_{\text {split }} \sim m_{\phi}$. The temperature of the thermal bath at that time, which we refer to as the maximum temperature $T_{\max }$, is given by

$$
T_{\max } \sim \alpha^{4 / 5} m_{\phi}\left(\frac{\Gamma_{\phi} M_{\mathrm{pl}}^{2}}{m_{\phi}^{3}}\right)^{2 / 5}
$$

After $t>t_{\text {split }}$, particles produced from the decay of the scalar field thermalize instantaneously, and eventually reheating is completed at $t_{\mathrm{rh}} \sim \Gamma_{\phi}^{-1}$. Note that the condition for the thermalization before reheating is completed is nothing but $t_{\mathrm{split}}<t_{\mathrm{rh}}$, which results in eq. (5.1). Relevant time scales are summarized in table 1.

\section{Conclusion and discussions}

In this paper, we have investigated the dynamics of reheating and thermalization of light particles which are produced from the perturbative decay of the scalar field, especially 


\begin{tabular}{|lcc|}
\hline time scale & general & $\operatorname{dim} 5$ \\
\hline$\left(m_{\phi} t\right)_{\text {soft th }}$ & $\alpha^{-3}\left(\frac{\Gamma_{\phi} M_{\mathrm{pl}}^{2}}{m_{\phi}^{3}}\right)^{-1 / 2}$ & $\alpha^{-3}$ \\
$\left(m_{\phi} t\right)_{\text {split }}$ & $\alpha^{-16 / 5}\left(\frac{\Gamma_{\phi} M_{\mathrm{pl}}^{2}}{m_{\phi}^{3}}\right)^{-3 / 5}$ & $\alpha^{-16 / 5}$ \\
$\left(m_{\phi} t\right)_{\mathrm{rh}}$ & $\left(\frac{\Gamma_{\phi}}{m_{\phi}}\right)^{-1}$ & $\frac{M_{\mathrm{pl}}^{2}}{m_{\phi}^{2}}$ \\
\hline
\end{tabular}

Table 1. Relevant time scales.

paying attention to the case with a small decay width of the scalar field, such as the one given by Planck-suppressed interactions. Since the number density is under occupied in this case, number violating splittings with small momentum exchanges and the evolution of the soft sector play crucial roles in the dynamics towards thermal equilibrium. Importantly, the bottleneck process is the dissipation of remaining hard particles into the soft sector as pointed out in refs. $[29,30]$ in the context of QGP.

Following their arguments, we have studied thermalization after/during reheating in detail, while paying attention to the red-shift of previously produced particles before the completion of reheating and to the dilution of their number density by the cosmic expansion. We have shown that thermalization takes place instantaneously in most cases, and also found that red-shifted previously produced particles are not relevant since radiated soft particles dominate the number density of red-shifted previously produced ones and that radiation is always dominated by that from hardest primaries which are not red-shifted. The condition under which produced particles thermalize before the completion of reheating is given by

$$
\alpha^{8 / 5} \gg \frac{m_{\phi}}{M_{\mathrm{pl}}}\left(\frac{\Gamma_{\phi} M_{\mathrm{pl}}^{2}}{m_{\phi}^{3}}\right)^{1 / 5},
$$

which turns out to be much milder than that obtained in the previous work [28] because the growth of the soft sector promotes the dissipation of remaining hard particles. As a result, even if the mass of the scalar field is as large as $10^{15} \mathrm{GeV}$, it is shown that thermalization takes place instantaneously in most cases. Our result is applicable to general situations where a decay of a non-relativistic matter reheats the universe via a small decay width and the decay products contain particles that are charged under a non-abalian gauge group.

Our analysis can also be applied to the instant preheating scenario [52] in which produced light particles have small number densities compared with thermal ones. The situation is quite similar to the case we have considered and hence our result is helpful in estimating the time scale when non-perturbative particle production is shut off by rescatterings $[53,54]$ (see also $[40,41]$ ). 


\section{Acknowledgments}

The authors thank Shigeki Matsumoto and Kazunori Nakayama for useful discussions. This work is supported by the World Premier International Research Center Initiative (WPI Initiative), MEXT, Japan (K.H.), and JSPS Research Fellowships for Young Scientists (K.H. and K.M.).

Open Access. This article is distributed under the terms of the Creative Commons Attribution License (CC-BY 4.0), which permits any use, distribution and reproduction in any medium, provided the original author(s) and source are credited.

\section{References}

[1] A.A. Penzias and R.W. Wilson, A measurement of excess antenna temperature at $4080 \mathrm{Mc} / \mathrm{s}$, Astrophys. J. 142 (1965) 419 [INSPIRE].

[2] J.C. Mather et al., Measurement of the cosmic microwave background spectrum by the COBE FIRAS instrument, Astrophys. J. 420 (1994) 439 [INSPIRE].

[3] D.N. Schramm and M.S. Turner, Big bang nucleosynthesis enters the precision era, Rev. Mod. Phys. 70 (1998) 303 [astro-ph/9706069] [INSPIRE].

[4] A.H. Guth, The inflationary universe: a possible solution to the horizon and flatness problems, Phys. Rev. D 23 (1981) 347 [INSPIRE].

[5] A.A. Starobinsky, A new type of isotropic cosmological models without singularity, Phys. Lett. B 91 (1980) 99 [inSPIRE].

[6] K. Sato, First order phase transition of a vacuum and expansion of the universe, Mon. Not. Roy. Astron. Soc. 195 (1981) 467 [INSPIRE].

[7] A.D. Linde and V.F. Mukhanov, Non-Gaussian isocurvature perturbations from inflation, Phys. Rev. D 56 (1997) 535 [astro-ph/9610219] [INSPIRE].

[8] K. Enqvist and M.S. Sloth, Adiabatic CMB perturbations in pre-big bang string cosmology, Nucl. Phys. B 626 (2002) 395 [hep-ph/0109214] [INSPIRE].

[9] D.H. Lyth and D. Wands, Generating the curvature perturbation without an inflaton, Phys. Lett. B 524 (2002) 5 [hep-ph/0110002] [INSPIRE].

[10] T. Moroi and T. Takahashi, Effects of cosmological moduli fields on cosmic microwave background, Phys. Lett. B 522 (2001) 215 [Erratum ibid. B 539 (2002) 303] [hep-ph/0110096] [INSPIRE].

[11] G.D. Coughlan, W. Fischler, E.W. Kolb, S. Raby and G.G. Ross, Cosmological problems for the Polonyi potential, Phys. Lett. B 131 (1983) 59 [INSPIRE].

[12] T. Banks, D.B. Kaplan and A.E. Nelson, Cosmological implications of dynamical supersymmetry breaking, Phys. Rev. D 49 (1994) 779 [hep-ph/9308292] [InSPIRE].

[13] B. de Carlos, J.A. Casas, F. Quevedo and E. Roulet, Model independent properties and cosmological implications of the dilaton and moduli sectors of $4 D$ strings, Phys. Lett. B 318 (1993) 447 [hep-ph/9308325] [INSPIRE].

[14] M. Fukugita and T. Yanagida, Baryogenesis without grand unification, Phys. Lett. B 174 (1986) 45 [INSPIRE]. 
[15] W. Buchmüller, P. Di Bari and M. Plümacher, Leptogenesis for pedestrians, Annals Phys. 315 (2005) 305 [hep-ph/0401240] [INSPIRE].

[16] M. Kawasaki and T. Moroi, Gravitino production in the inflationary universe and the effects on big bang nucleosynthesis, Prog. Theor. Phys. 93 (1995) 879 [hep-ph/9403364] [INSPIRE].

[17] M. Kawasaki, K. Kohri, T. Moroi and A. Yotsuyanagi, Big-bang nucleosynthesis and gravitino, Phys. Rev. D 78 (2008) 065011 [arXiv:0804.3745] [INSPIRE].

[18] I. Affleck and M. Dine, A new mechanism for baryogenesis, Nucl. Phys. B 249 (1985) 361 [INSPIRE].

[19] M. Dine, L. Randall and S.D. Thomas, Baryogenesis from flat directions of the supersymmetric standard model, Nucl. Phys. B 458 (1996) 291 [hep-ph/9507453] [INSPIRE].

[20] R. Allahverdi, B.A. Campbell and J.R. Ellis, Reheating and supersymmetric flat direction baryogenesis, Nucl. Phys. B 579 (2000) 355 [hep-ph/0001122] [INSPIRE].

[21] A. Anisimov and M. Dine, Some issues in flat direction baryogenesis, Nucl. Phys. B 619 (2001) 729 [hep-ph/0008058] [INSPIRE].

[22] M. Fujii, K. Hamaguchi and T. Yanagida, Reheating temperature independence of cosmological baryon asymmetry in Affleck-Dine leptogenesis, Phys. Rev. D 63 (2001) 123513 [hep-ph/0102187] [INSPIRE].

[23] J.R. Ellis, K. Enqvist, D.V. Nanopoulos and K.A. Olive, Inflationary fluctuations, entropy generation and baryogenesis, Phys. Lett. B 191 (1987) 343 [inSPIRE].

[24] J. McDonald, Reheating temperature and inflaton mass bounds from thermalization after inflation, Phys. Rev. D 61 (2000) 083513 [hep-ph/9909467] [INSPIRE].

[25] R. Allahverdi, Thermalization after inflation and reheating temperature, Phys. Rev. D 62 (2000) 063509 [hep-ph/0004035] [INSPIRE].

[26] A. Mazumdar and B. Zaldivar, Quantifying the reheating temperature of the universe, arXiv: 1310.5143 [INSPIRE].

[27] D.J.H. Chung, E.W. Kolb and A. Riotto, Production of massive particles during reheating, Phys. Rev. D 60 (1999) 063504 [hep-ph/9809453] [INSPIRE].

[28] S. Davidson and S. Sarkar, Thermalization after inflation, JHEP 11 (2000) 012 [hep-ph/0009078] [INSPIRE].

[29] R. Baier, A.H. Mueller, D. Schiff and D.T. Son, 'Bottom up' thermalization in heavy ion collisions, Phys. Lett. B 502 (2001) 51 [hep-ph/0009237] [INSPIRE].

[30] A. Kurkela and G.D. Moore, Thermalization in weakly coupled nonabelian plasmas, JHEP 12 (2011) 044 [arXiv:1107.5050] [INSPIRE].

[31] S. Dimopoulos, G.R. Dvali and R. Rattazzi, Dynamical inflation and unification scale on quantum moduli spaces, Phys. Lett. B 410 (1997) 119 [hep-ph/9705348] [INSPIRE].

[32] F. Takahashi, Linear inflation from running kinetic term in supergravity, Phys. Lett. B 693 (2010) 140 [arXiv: 1006.2801] [InSPIRE].

[33] K. Nakayama and F. Takahashi, Running kinetic inflation, JCAP 11 (2010) 009 [arXiv: 1008.2956] [INSPIRE].

[34] K. Harigaya, M. Ibe, K. Schmitz and T.T. Yanagida, Chaotic inflation with a fractional power-law potential in strongly coupled gauge theories, Phys. Lett. B 720 (2013) 125 [arXiv: 1211.6241] [INSPIRE]. 
[35] J. Yokoyama, Fate of oscillating scalar fields in the thermal bath and their cosmological implications, Phys. Rev. D 70 (2004) 103511 [hep-ph/0406072] [INSPIRE].

[36] J. Yokoyama, Can oscillating scalar fields decay into particles with a large thermal mass?, Phys. Lett. B 635 (2006) 66 [hep-ph/0510091] [INSPIRE].

[37] M. Bastero-Gil, A. Berera and R.O. Ramos, Dissipation coefficients from scalar and fermion quantum field interactions, JCAP 09 (2011) 033 [arXiv: 1008.1929] [INSPIRE].

[38] M. Bastero-Gil, A. Berera, R.O. Ramos and J.G. Rosa, General dissipation coefficient in low-temperature warm inflation, JCAP 01 (2013) 016 [arXiv:1207.0445] [INSPIRE].

[39] M. Drewes, On the role of quasiparticles and thermal masses in nonequilibrium processes in a plasma, arXiv:1012.5380 [INSPIRE].

[40] K. Mukaida and K. Nakayama, Dynamics of oscillating scalar field in thermal environment, JCAP 01 (2013) 017 [arXiv: 1208.3399] [INSPIRE].

[41] K. Mukaida, K. Nakayama and M. Takimoto, Fate of $Z_{2}$ symmetric scalar field, JHEP 12 (2013) 053 [arXiv: 1308.4394] [INSPIRE].

[42] K. Mukaida and K. Nakayama, Dissipative effects on reheating after inflation, JCAP 03 (2013) 002 [arXiv: 1212.4985] [INSPIRE].

[43] M. Drewes and J.U. Kang, The kinematics of cosmic reheating, Nucl. Phys. B 875 (2013) 315 [arXiv:1305.0267] [INSPIRE].

[44] P.B. Arnold, G.D. Moore and L.G. Yaffe, Effective kinetic theory for high temperature gauge theories, JHEP 01 (2003) 030 [hep-ph/0209353] [INSPIRE].

[45] L.D. Landau and I. Pomeranchuk, Limits of applicability of the theory of bremsstrahlung electrons and pair production at high-energies, Dokl. Akad. Nauk Ser. Fiz. 92 (1953) 535 [INSPIRE].

[46] A.B. Migdal, Bremsstrahlung and pair production in condensed media at high-energies, Phys. Rev. 103 (1956) 1811 [INSPIRE].

[47] P.B. Arnold, G.D. Moore and L.G. Yaffe, Photon emission from ultrarelativistic plasmas, JHEP 11 (2001) 057 [hep-ph/0109064] [INSPIRE].

[48] P.B. Arnold, G.D. Moore and L.G. Yaffe, Photon emission from quark gluon plasma: complete leading order results, JHEP 12 (2001) 009 [hep-ph/0111107] [INSPIRE].

[49] P.B. Arnold, G.D. Moore and L.G. Yaffe, Photon and gluon emission in relativistic plasmas, JHEP 06 (2002) 030 [hep-ph/0204343] [INSPIRE].

[50] D. Besak and D. Bödeker, Hard thermal loops for soft or collinear external momenta, JHEP 05 (2010) 007 [arXiv: 1002.0022] [INSPIRE].

[51] K. Harigaya and K. Mukaida, in preparation.

[52] G.N. Felder, L. Kofman and A.D. Linde, Instant preheating, Phys. Rev. D 59 (1999) 123523 [hep-ph/9812289] [INSPIRE].

[53] L. Kofman, A.D. Linde and A.A. Starobinsky, Reheating after inflation, Phys. Rev. Lett. 73 (1994) 3195 [hep-th/9405187] [INSPIRE].

[54] L. Kofman, A.D. Linde and A.A. Starobinsky, Towards the theory of reheating after inflation, Phys. Rev. D 56 (1997) 3258 [hep-ph/9704452] [INSPIRE]. 